Pesq. Vet. Bras. 36(12):1145-1154, dezembro 2016 DOI: 10.1590/S0100-736X2016001200001

\title{
Epidemiologia descritiva da raiva dos herbívoros notificados no estado do Paraná entre 1977 e $2012^{1}$
}

\author{
Romerson Dognani ${ }^{2 *}$, Elzira J. Pierre ${ }^{2}$, Maria do Carmo P. Silva ${ }^{2}$, Maria A.C. Patrício ${ }^{3}$, \\ Silvano C. da Costa ${ }^{4}$, Jair R. do Prado ${ }^{4}$ e Júlio A.N. Lisbôa ${ }^{5 *}$
}

\begin{abstract}
Dognani R., Pierre E.J., Silva M.C.P., Patrício M.A.C., Costa S.C., Prado J.R. \& Lisbôa J.A.N. 2016. [Epidemiology of rabies in large animals of Parana, Brazil, from 1977 to 2012.] Epidemiologia descritiva da raiva dos herbívoros notificados no estado do Paraná entre 1977 e 2012. Pesquisa Veterinária Brasileira 36(12):1145-1154. Departamento de Clínicas Veterinárias, Centro de Ciências Agrárias, Universidade Estadual de Londrina, Campus Universitário, Caixa Postal 10011, Londrina, PR 86057-970, Brazil. E-mail: janlisboa@uel.br

A descriptive epidemiological survey of rabies in herbivorous reared in the state of Parana, Brazil, was carried out from 1977 to 2012. The positive cases and the total number of brain samples processed for diagnostic purposes were distributed according to species, year, month, geographical region and municipality. Chi-square test was used to verify if rabies was associated to these factors. Probabilistic model was applied to historical series in order to verify cyclic and seasonal patterns. In Parana, rabies is an endemic disease with variable yearly occurrence, without seasonal pattern and with a possible cyclic pattern every 18 years. Cattle $(86.9 \%)$ and equides $(11.3 \%)$ were mainly affected. Rabies was registered in $47.6 \%$ of all municipalities, indicating a great spread of this disease in Parana, mainly during the last decade. Middlewest and Curitiba regions, followed by Pioneer North and West regions, were the areas of most occurrence. The number of cases per municipality was weakly correlated with the number of shelters for Desmodus rotundus ( $\mathrm{r}=0.469 ; \mathrm{p}<0.0001)$. Therefore, we suggest that annual immunization of cattle and equides should be applied in the high occurrence areas (Middlewest and Curitiba regions) and encouraged in intermediate occurrence areas (Pioneer North and West regions).
\end{abstract}

INDEX TERMS: Epidemiology, rabies, Parana, Brazil, Lyssavirus, encephalitis, cattle, equids, mortality.

RESUMO-- Realizou-se estudo epidemiológico descritivo da raiva dos herbívoros no estado do Paraná entre os anos de 1977 e 2012. Os casos confirmados de raiva e o total de amostras de encéfalo encaminhadas para o diag-

\footnotetext{
${ }^{1}$ Recebido em 14 de junho de 2015.

Aceito para publicação em 4 de fevereiro de 2016.

Parte da Dissertação de Mestrado em Ciência Animal do primeiro autor, Universidade Estadual de Londrina (UEL).

${ }^{2}$ Médico Veterinário da Agência de Defesa Agropecuária do Paraná (ADAPAR), Rua dos Funcionários 1559, Curitiba, PR 80035-050, Brasil.

${ }^{3}$ Centro de Diagnóstico Marcos Enrietti (CDME), Rua Jaime Balão 575, Curitiba, PR 80040-340, Brasil.

${ }^{4}$ Departamento de Estatística, Centro de Ciências Exatas, UEL, Campus Universitário, Cx. Postal 10011, Londrina, PR 86057-970, Brasil.

${ }^{5}$ Departamento de Clínicas Veterinárias, Centro de Ciências Agrárias, UEL, Campus Universitário, Cx. Postal 10011, Londrina, PR 86057-970, Brasil. *Autor para correspondência: janlisboa@uel.br
}

nóstico foram distribuídos por espécie, por ano, por meses, por mesorregião geográfica e por municípios, aplicando-se o teste de Qui-quadrado para verificar se havia associação com esses fatores. Modelo probabilístico foi ajustado à série histórica para verificação de padrões cíclico ou sazonal. Comprovou-se que a raiva é uma doença endêmica no PR, com ocorrência variável entre os anos, sem padrão sazonal e com ciclicidade aparente a cada 18 anos, acometendo, sobretudo, bovinos $(86,9 \%)$ e equídeos $(11,3 \%)$. Verificou-se grande difusão no estado (47,6\% dos municípios), e a maior expansão geográfica aconteceu na última década. As áreas de ocorrência maior foram as mesorregiões Centro Oriental e de Curitiba, seguidas por Norte Pioneiro e Oeste. 0 número de casos de raiva por município se correlacionou, ainda que fracamente, com o número de abrigos de Desmodus rotundus $(\mathrm{r}=0,469 ; \mathrm{p}<0,0001)$. Sugere-se que a imunização anual de bovinos e equídeos passe a ser ado- 
tada nas áreas de maior ocorrência (mesorregiões Centro Oriental e de Curitiba) e encorajada nas de ocorrência intermediária (mesorregiões Norte Pioneiro e Oeste).

TERMOS DE INDEXAÇÃO: Epidemiologia, raiva, Paraná, Lyssavirus, encefalite, bovinos, equídeos, mortalidade.

\section{INTRODUÇÃO}

A raiva, doença caracterizada por encefalite progressiva fatal e causada por um vírus do gênero Lyssavirus, é conhecida desde a antiguidade e ainda possui grande relevância nos dias de hoje, provocando a morte de animais pecuários, selvagens e domésticos de estimação, e trazendo risco extremo à saúde humana (Brasil 2009). Do ponto de vista epidemiológico, a enfermidade distingue-se em dois ciclos: urbano e silvestre (Acha \& Szyfres 2003). No ciclo silvestre, a infecção do homem é menos provável (Wada et al. 2011) e diferentes espécies de morcegos e outros animais selvagens podem ser reservatórios do vírus, sendo Desmodus rotundus o principal responsável pela transmissão da infecção e os herbívoros domésticos os principais animais acometidos (Rodriguez et al. 2007).

A raiva nos herbívoros ocorre em todo o território nacional, e, embora a sua incidência varie conforme a região geográfica, de modo geral pode ser considerada endêmica no país (Brasil 2013). O Programa Nacional de Controle da Raiva dos Herbívoros, coordenado pelo Ministério da Agricultura, Pecuária e Abastecimento (MAPA) e executado em cada estado pelo órgão respectivo responsável pela Defesa Sanitária Animal, existe desde 1966 sendo regulamentado em 1976 (Brasil 1976) e atualizado em 2002 (Brasil 2002). As ações de vigilância epidemiológica que compreendem atendimento a casos suspeitos, colheita de material e confirmação do diagnóstico não sofreram alteração sistemática ao longo do tempo, de acordo com a legislação.

Estudos sobre a ocorrência da doença foram realizados em diferentes estados, abrangendo observações em intervalos de tempo (sequência de anos) maiores do que 20 anos (Silva et al. 2001a, Santos et al. 2006, Teixeira et al. 2008) ou menores do que 15 anos (Feital \& Confalonieri 1998, Neves 2008, Galiza et al. 2010, Matta et al. 2010, Gomes \& Monteiro 2011, Oliveira et al. 2012), e apontaram aspectos epidemiológicos diversos, caracterizando sazonalidade, ciclicidade, regiões de risco maior, espécies acometidas, entre outros.

No estado do Paraná, a raiva em herbívoros é reconhecida desde 1927 (Schlögel 1985), mas poucos levantamentos sobre a doença foram realizados. A ocorrência em espécies herbívoras e carnívoras foi investigada entre 1955 e 1989, mas as observações se limitaram à região de Curitiba (Monteiro et al. 1992). Montaño et al. (1987), por outro lado, estudaram os casos de raiva que ocorreram em bovinos de todo o estado, mas limitaram o período de observação entre 1974 e 1984. Mais recentemente, a ocorrência em ruminantes (bovinos, ovinos e caprinos) foi investigada de 2004 a 2006 (Patrício et al. 2007) e em 2007 (Patrício et al. 2009). Com a finalidade de contribuir com a caracterização epidemiológica mais abrangente da raiva dos herbívoros no estado do Paraná, esse trabalho foi desenvolvido consi- derando informações sobre a ocorrência da doença em 36 anos seguidos (1977-2012).

\section{MATERIAL E MÉTODOS}

Realizou-se um estudo observacional descritivo retrospectivo, utilizando registros dos casos de raiva diagnosticados em herbívoros domésticos no estado do Paraná, entre os anos de 1977 e 2012. Os dados foram fornecidos pela Agência de Defesa Agropecuária do Paraná (ADAPAR), vinculada à Secretaria do Estado da Agricultura e do Abastecimento do Paraná (SEAB-PR), e levantados junto às Áreas de Epidemiologia e de Raiva dos Herbívoros, sendo a última responsável pela coordenação do Programa Estadual de Controle e Profilaxia da Raiva dos Herbívoros.

O Centro de Diagnóstico Marcos Enrietti (CDME) e o Laboratório Central do Estado do Paraná (LACEN-PR), localizados em Curitiba, possuem os dois únicos laboratórios que realizam o diagnóstico de raiva no estado. O CDME, integrante da estrutura da SEAB-PR, possui o laboratório, credenciado pelo MAPA, para o qual são encaminhadas as amostras provenientes de animais pecuários. Todas as amostras do Sistema Nervoso Central dos herbívoros com sinais de doença neurológica, colhidas por médicos veterinários autônomos, do Serviço Oficial de Defesa Sanitária Animal e das Instituições de Ensino Superior paranaenses, foram, preferencialmente, encaminhadas e processadas nesse mesmo laboratório, durante todo o período de abrangência do estudo. 0 LACEN-PR recebeu e processou raramente, amostras de herbívoros, e esses resultados também foram computados no presente estudo. As amostras encaminhadas consistiram, mais comumente, em fragmentos do encéfalo e, menos frequentemente, no encéfalo como um todo, incluindo o tronco cerebral e o início da medula cervical. 0 diagnóstico foi confirmado por meio da técnica de imunofluorescência direta (IFD), complementada pela prova biológica (inoculação intracerebral em camundongos), de acordo com o recomendado pela Organização Mundial para a Saúde Animal (Office International des Epizooties - OIE 2014). Os dois procedimentos para a confirmação do diagnóstico foram empregados em todas as amostras examinadas.

Considerando-se o número de amostras processadas e o número de casos de raiva diagnosticados, realizou-se análise exploratória dos dados de distribuição por ano, por meses do ano, por espécie acometida, por município e por mesorregião geográfica. As espécies equina, muar e asinina foram agrupadas como equídeos. Devido à intensa variabilidade da ocorrência entre os 36 anos estudados, o limiar endêmico foi calculado de duas maneiras: (a) usando a mediana dos casos de raiva por ano, e definindo os limites endêmicos inferior e superior como o primeiro e o terceiro quartis, respectivamente (Medronho et al. 2009); e (b) aplicando a média móvel simples e seus respectivos desvios-padrão anuais como indicadores dos limites endêmicos superior e inferior.

A análise de decomposição e modelagem da série histórica foi realizada empregando-se métodos recomendados por Morettin \& Toloi (2006). Primeiramente, foi realizada a transformação logarítmica dos dados originais com o intuito de estabilizar a variância e de tornar os efeitos sazonal e/ou cíclico aditivos. Para verificar a existência de ciclicidade e/ou de sazonalidade foi utilizado, preliminarmente, o teste de Fisher. Para verificação da existência de tendência empregou-se o teste do sinal (Cox-Stuart). Após a identificação dos componentes de tendência, sazonalidade e ciclicidade, ajustaram-se os modelos de Box e Jenkins, separadamente para os casos de raiva e para os casos de outras doenças, com a finalidade de caracterizar o comportamento das séries históricas e de fazer previsão. Após a construção dos modelos utilizou-se o teste de Ljung-Box para verificar a adequação dos mesmos, ou 
seja, se os resíduos estavam não correlacionados (ruído branco). Empregou-se o programa R para a realização das análises e admitiu-se o nível de significância dos testes de 5\%.

As informações sobre a localização e o tipo dos abrigos dos morcegos transmissores foram obtidas das fichas de controle de cadastro de abrigos de quirópteros hematófagos do ano de 2011, mantidas pela Área de Raiva dos Herbívoros da ADAPAR. Para mapear a distribuição espacial dos casos de raiva e dos abrigos cadastrados foi utilizado o programa TABWIN 2.0, considerando-se os municípios e as mesorregiões paranaenses.

Os casos de raiva foram apresentados com valores absolutos e os valores relativos foram calculados para a taxa de positividade, a distribuição entre as espécies e a difusão entre os municípios. 0 teste do Qui-quadrado foi empregado para investigar se a ocorrência da raiva estava associada com os meses do ano, com a espécie acometida e com as mesorregiões geográficas. 0 teste de correlação de Pearson foi empregado para verificar a existência de correlações entre o número de casos confirmados, o número de amostras enviadas e a taxa de positividade anuais. Para o estudo da correlação entre o número de casos de raiva e o número de abrigos de morcegos hematófagos cadastrados, por município e por mesorregião geográfica, consideraram-se somente as informações dos últimos seis anos (2007-2012), respeitando a contemporaneidade com a informação disponível sobre a localização dos abrigos. Empregou-se o programa SigmaStat 3.1 para a realização das análises estatísticas, admitindo-se a probabilidade de erro de $5 \%$.

\section{RESULTADOS E DISCUSSÃO}

Durante os 36 anos estudados, 9.546 amostras de encéfalos de herbívoros foram processadas para o diagnóstico de raiva, e a doença foi confirmada em 2.682 animais (taxa de positividade geral de 28,1\%) (Quadro 1). 0 número de amostras analisadas $(265 \pm 141$ amostras por ano) indica que as enfermidades que cursam com sinais clínicos neurológicos, particularmente em bovinos e em equídeos, são frequentes no estado e o diagnóstico diferencial para a raiva é indispensável. A taxa de positividade variou entre os anos $(2,9-43,0 \%)$ com valor médio de $26,1 \pm 9,4 \%$ e mediano de $26,7 \%$. Outros pesquisadores verificaram taxas de positividade semelhantes e até maiores do que as encontradas neste trabalho. No estudo realizado por Monteiro et al. (1992), na região metropolitana de Curitiba, verificou-se taxa de positividade de $32,1 \%$ para a raiva em herbívoros. Em MS (Neves 2008), nos estados da Região Norte (Casseb et al. 2006), em MT (Matta et al. 2010) e no RS (Teixeira et al. 2008) as taxas foram, respectivamente, $23,8 \%, 30,5 \%$, $33,5 \%$ e 33,9\%. Em Minas Gerais as taxas foram mais elevadas, variando entre 50,5\% de 1976 até 1997 (Silva et al.

\section{Quadro 1. Amostras de encéfalo de herbívoros enviadas para diagnóstico, casos confirmados de raiva e taxas de positividade separados por espécie, durante o período de 1977 a 2012, no Paraná}

\begin{tabular}{lccc}
\hline Espécie de herbívoro & $\begin{array}{c}\text { Amostras } \\
\text { enviadas }\end{array}$ & $\begin{array}{c}\text { Casos de } \\
\text { raiva }\end{array}$ & $\begin{array}{c}\text { Taxa de } \\
\text { positividade (\%) }\end{array}$ \\
\hline Bovinos & 7627 & 2331 & 30,6 \\
Equídeos & 1411 & 303 & 21,5 \\
Ovinos & 300 & 19 & 6,3 \\
Bubalinos & 73 & 17 & 23,3 \\
Caprinos & 135 & 12 & 8,9 \\
TOTAL & 9546 & 2682 & 28,1
\end{tabular}

2001a), 44,4\% até 2006 (Menezes et al. 2008) e 41,5\% até 2010 (Oliveira et al. 2012). A taxa de positividade mais reduzida foi verificada na região noroeste de SP, onde a raiva foi confirmada em somente $12,9 \%$ dos casos (Queiroz et al. 2009). Esses resultados indicam, de forma geral, que a raiva deve ser considerada a doença neurológica de maior importância nos herbívoros, especialmente nos bovinos e nos equídeos.

Considerando as taxas de positividade (Quadro 1), seria possível admitir, como base diagnóstica, que de cada 10 bovinos paranaenses que morrerem acometidos por doença neurológica, três possam ser raivosos, e de cada 10 equídeos, dois podem ter a doença. Essa estimativa pode servir somente como informação para o médico veterinário no momento da formulação das hipóteses diagnósticas frente a casos de doença do Sistema Nervoso Central (SNC) em bovinos e em equídeos. A estimativa não se aplica às demais espécies de herbívoros porque o número de observações foi comparativamente menor. Nos estudos de casuística das doenças neurológicas realizados no Brasil, a raiva sempre é apontada como a principal enfermidade do SNC nos bovinos (Sanches et al. 2000, Galiza et al. 2010, Ribas et al. 2013) e uma das principais nos equídeos (Lima et al. 2005, Pimentel et al. 2009). 0 mesmo não é verdadeiro para os ovinos e caprinos, nos quais a doença é observada em número pequeno de casos (Lima et al. 2005, Guedes et al. 2007, Rissi et al. 2010). Também no PR, estudo prospectivo sobre o diagnóstico diferencial de doenças neurológicas em 136 bovinos, realizado entre 2009 e 2012, revelou que $38 \%$ dos animais $(n=51)$ apresentavam encefalite, causada principalmente pelo vírus da raiva $(\mathrm{n}=16)$ e por BoHV-5 ( $\mathrm{n}=20$ ) (Queiroz et al. 2013).

Levando em consideração os valores de média e da mediana, a frequência absoluta da raiva no período estudado foi de 74 ou 67 casos por ano, respectivamente. Entretanto, fica evidente que o número de casos de raiva por ano apresentou grande variabilidade durante o período de abrangência do estudo (Fig.1). Em 14 dos 36 anos do levantamento o número de casos foi próximo da mediana, em 10 anos o número foi superior à mesma e nos 12 restantes a ocorrência foi reduzida. Ao se definirem os limites endêmicos inferior (25 casos) e superior (107 casos) como o primeiro e o terceiro quartis, respectivamente, pode-se afirmar que a doença é endêmica no estado, pois a ocorrência se situou entre esses limites em 21 anos. Somente em sete anos o número de casos extrapolou o limite superior podendo caracterizar epidemia (anos de 1984, 1985, 1987, 1993, 2006, 2007 e 2009). Em dois períodos definidos (1977-1980 e 1998-2001), ao contrário, registraram-se tão poucos casos da enfermidade que, nesses anos, seria possível classificar a ocorrência como sob controle no Paraná. Por outro lado, quando os limites endêmicos inferior e superior são definidos como os desvios-padrão das médias móveis a cada ano, confirma-se que a doença manteve-se endêmica no estado, pois o número de casos se situou entre esses limites durante todo o período.

A taxa de positividade teve, também, grande variação durante o período do estudo, seguindo padrão pareci- 
do com o da ocorrência da raiva. Segundo Queiroz et al. (2009), a taxa de positividade reflete, de certa forma, a situação epidemiológica da doença em determinada região. As menores taxas de positividade $(12,1 \%$ em $1998,2,9 \%$ em 1999 e $12,8 \%$ em 2000) foram observadas em anos em que o número de casos de raiva foi reduzido. Da mesma manei$\mathrm{ra}$, as maiores taxas de positividade foram verificadas nos anos em que a ocorrência da raiva foi maior (entre 38,2\% e $42,2 \%$ em 1984, 2006, 2007 e 2009). De fato, a taxa de positividade apresentou correlação forte com o número de casos de raiva diagnosticado a cada ano $(\mathrm{r}=0,707 ; \mathrm{p}<0,001)$.

0 número de amostras enviadas anualmente para diagnóstico também variou durante os 36 anos, e essa variação foi parecida com a da ocorrência da raiva $(r=0,895$; $\mathrm{p}<0,001)$. Na maioria do período, com exceção dos quatro primeiros anos, o número de amostras submetidas a diagnóstico manteve-se acima de 100 amostras/ano, o que indica que o trabalho de vigilância para a enfermidade manteve-se ativo durante todo o tempo no estado.

$\mathrm{Na}$ análise da série histórica dos casos de raiva constataram-se as ausências de tendência $(p>0,05)$ e de sazonalidade, porém a existência de ciclicidade a cada 18 anos. 0 modelo ajustado SARIMA $(1,0,0) \times(0,1,0)$ pode ser descrito pela equação $Z_{t}=\exp \left[a_{t} /(1-0,5653 B)\left(1-B^{18}\right) ;\right.$ onde $Z_{t}$ é o conjunto de observações da série temporal de raiva, $a_{t}$ é o componente aleatório e $B_{t}$ é o operador de retardo, tal que $B^{m} Z_{t}=Z_{t-m^{*}}$. Na análise da série histórica dos casos de outras doenças neurológicas constataram-se as ausências de tendência $(p>0,05)$ e de sazonalidade, porém a existência de ciclicidade a cada 18 anos, tal como observado para os casos de raiva. 0 modelo ajustado SARIMA $(1,0,0) \times(0,1,0)_{18}$ pode ser descrito pela equação $Y_{t}=\exp \left[a_{t} /(1-0,9078 B)\right.$ (1- $\left.\left.B^{18}\right)\right]$; onde $Y_{t}$ é o conjunto de observações da série temporal de outras doenças neurológicas, $a_{t}$ é o componente aleatório e $B_{t}$ é o operador de retardo, tal que $B^{m} Y_{t}=Y_{t-m^{*}}$. As Figuras 2 e 3 demonstram que os modelos ajustados foram satisfatórios para descrever o comportamento das variáveis, ou seja, os valores estimados se aproximaram dos valores observados. Usando o modelo ajustado para realizar previsões com o nível de confiança de 95\%, o esperado para o ano de 2014 seria de 48 a 58 casos de raiva e de 165 a 173 casos de outras doenças neurológicas. De fato ocorreram 40 casos confirmados de raiva e 138 casos de outras doenças neurológicas em herbívoros no estado em 2014. Isso indica que a previsão, com base no modelo ajustado, é coerente e confirma que o número de casos de raiva encontra-se na curva descendente. Se a previsão é correta, provavelmente, o ano de 2017 voltará a registrar número muito reduzido de casos da doença no estado.

0 resultado de ciclicidade sugerida a cada 18 anos contraria um conceito comum de que a raiva ocorra de forma cíclica a cada cinco ou sete anos, como apontado no MS (Mori \& Lemos 1998, Neves 2008, Ribas et al. 2013), na PB (Galiza et al. 2010) e em SP (Gomes \& Monteiro 2011). Nos estudos retrospectivos que abrangeram períodos maiores de observação, a ciclicidade da raiva nunca foi confirmada, seja no RJ (Feital \& Confalonieri 1998), em MG (Silva et al. 2001a, Menezes et al. 2008), em GO (Santos et al. 2006), no RS (Teixeira et al. 2008), ou em MT (Matta et al. 2010).
Os dados oficiais de ocorrência da doença disponíveis no site do Ministério da Agricultura, Pecuária e do Abastecimento abrangendo o período entre 1996 e 2010 (Brasil 2013) também não sustentam que exista este comportamento. Com base no conjunto de resultados dos estudos, seria correto admitir que a ocorrência da doença é variável entre os anos, mas a ciclicidade da raiva parece ser questionável. No PR, contrariamente à situação de alguns estados brasileiros, o possível comportamento cíclico da doença foi

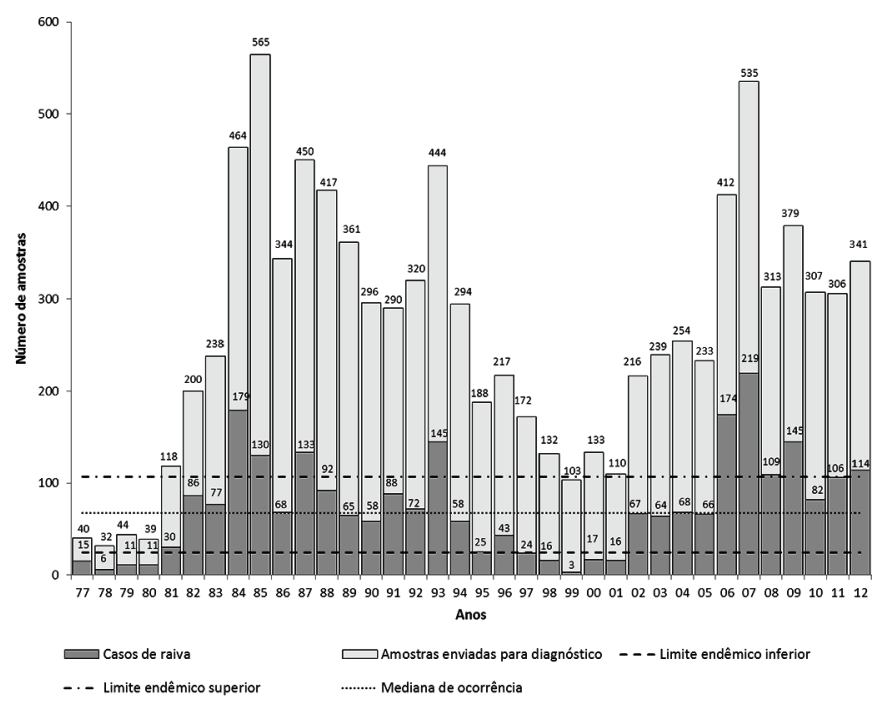

Fig.1. Amostras de encéfalos de herbívoros encaminhadas para diagnóstico e limiar endêmico da raiva dos herbívoros no estado do Paraná, durante o período de 1977 a 2012.

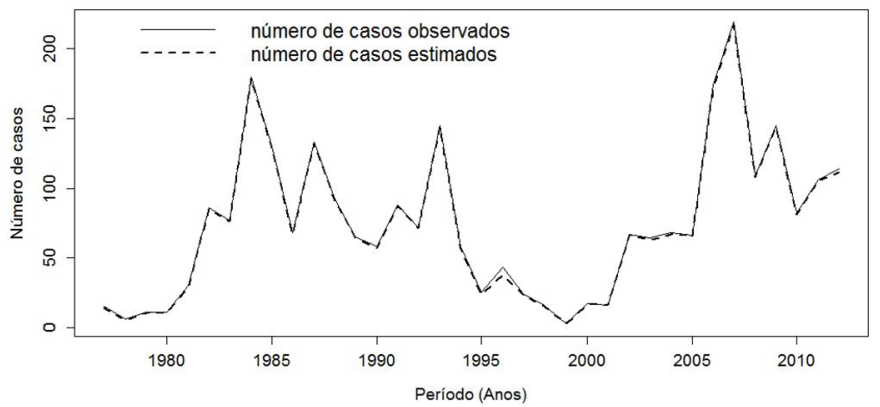

Fig.2. Séries de valores observados e estimados de acordo com o modelo ajustado SARIMA $(1,0,0) \times(0,1,0)_{18}$, para os casos de raiva em herbívoros entre 1977 e 2012, no Paraná.

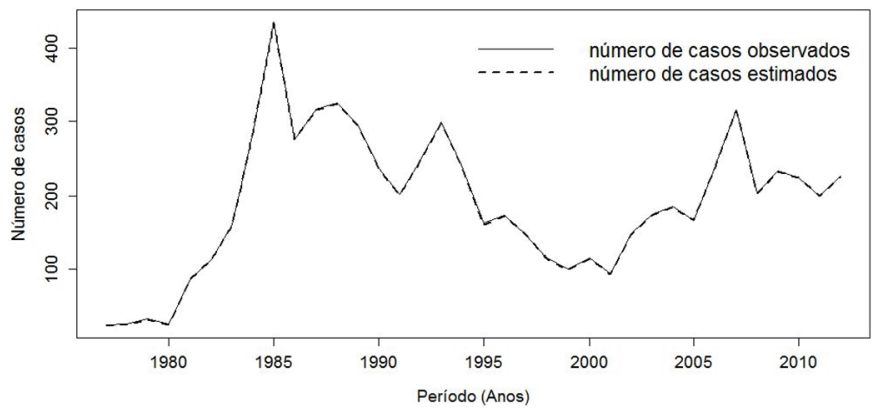

Fig.3. Séries de valores observados e estimados de acordo com o modelo ajustado SARIMA $(1,0,0) \times(0,1,0)_{18}$, para os casos de outras doenças neurológicas em herbívoros entre 1977 e 2012, no Paraná. 
sugerido no presente estudo. Os motivos que determinam o ciclo de 18 anos não são claros, entretanto, e precisariam ser elucidados em investigações futuras.

A ocorrência da raiva se diferenciou entre as espécies $(\mathrm{p}<0,001)$, sendo mais comum em bovinos e em equídeos (Quadro 1 e Fig.4). A maioria das amostras enviadas foi de bovinos (7.627) e, consequentemente, o maior número de casos foi confirmado nessa espécie (2.331). Os equídeos ocuparam o segundo lugar, tanto de amostras enviadas quanto de casos de raiva diagnosticados. A ocorrência em bubalinos e nos pequenos ruminantes foi reduzida. Essa mesma relação de frequência entre as espécies é observada nos demais estados brasileiros ao longo dos anos (Brasil 2013), caracterizando um padrão de ocorrência da enfermidade. Quando comparados, em valores relativos, a ocorrência entre as espécies e a distribuição dos rebanhos de herbívoros domésticos no PR (Fig.4), verifica-se que, no caso da espécie bovina, a ocorrência maior deve estar associada ao maior tamanho do rebanho dessa espécie suscetível. 0 mesmo não se pode afirmar dos equídeos, para os quais os percentuais de amostras de encéfalos processadas $(14,7 \%)$ e de casos de raiva confirmados $(11,3 \%)$ foram discrepantes e mais elevados do que o percentual de equídeos dentre os herbívoros criados no estado $(5,03 \%)$. Isso sugere que a raiva seja relativamente mais incidente nos equídeos, o que pode ser justificado pela preferência do Desmodus rotundus em se alimentar nos equídeos em

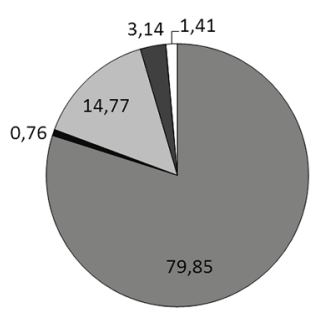

B

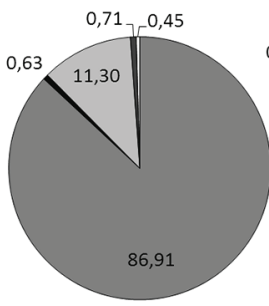



$\square$ Bovinos $\square$ Bubalinos $\square$ Equídeos $\square$ Ovinos $\square$ Caprinos

Fig.4. (A) Distribuição percentual, entre os herbívoros, das amostras de encéfalo enviadas para diagnóstico e (B) dos casos de raiva confirmados no Paraná de 1977 a 2012; (C) além das médias dos rebanhos paranaenses entre 1980 e 2011 (Paraná 2013). comparação a outros herbívoros domésticos (Arruda et al. 2013).

Ao contrário dos equídeos a raiva foi relativamente menos incidente nos pequenos ruminantes, porque o percentual de ovinos e de caprinos raivosos foi comparativamente menor do que o percentual dessas populações no rebanho paranaense de herbívoros domésticos (Fig.4). Provavelmente, isto se justifica pela menor frequência de ataque de morcegos hematófagos a estas espécies em comparação aos bovinos e equídeos (Arruda et al. 2013).

Quanto aos meses do ano, embora a associação tenha se comprovado significativa $(\mathrm{p}<0,01)$, a raiva não ocorreu obedecendo a um padrão definido de sazonalidade, e o mesmo se verificou para o caso das outras doenças neurológicas. Nos estados do RJ (Feital \& Confalonieri 1998), MG (Silva et al. 2001a, Menezes et al. 2008), MT (Matta et al. 2010) e RS (Marcolongo-Pereira et al. 2011), os resultados foram diferentes desse, pois houve maior concentração dos casos nos meses de outono e de inverno. 0 motivo do aumento de casos de raiva no período do inverno, tradicionalmente frio e seco, pode estar relacionado com hábitos do morcego hematófago transmissor do vírus, sendo essa a época do ano preferencial para o seu acasalamento (Aguiar 2007). 0 fato de esses quirópteros evitarem abandonar os seus abrigos nos dias chuvosos (Uieda 1982) também pode contribuir para diminuir a chance de infecção dos herbívoros nos meses em que a chuva é mais frequente. Contrariando as evidências desses estudos, Gomes \& Monteiro (2011) também não observaram padrão de sazonalidade da raiva em SP, e Neves (2008) verificou que a raiva é mais frequente nos meses do verão e do outono em MS.

Na distribuição geográfica da raiva dos herbívoros, verificou-se que a doença foi difundida pelo estado (Quadro 2), atingindo $47,6 \%$ dos municípios durante o período. Esteve presente em todas as mesorregiões geográficas do estado, porém, em diferentes graus de ocorrência $(p<0,001)$; concentrando-se em certas áreas do Paraná. Nas mesorregiões Centro Oriental e de Curitiba esteve mais difundida, registrando-se casos da doença em $100 \%$ e $86,49 \%$ dos municípios, respectivamente. Na mesorregião Centro Ocidental, por outro lado, casos da doença foram registrados em apenas $8 \%$ dos municípios. A taxa de positividade também variou de acordo com a mesorregião geográfica,

Quadro 2. Difusão da raiva dos herbívoros, número de amostras de encéfalo colhidas para diagnóstico, casos confirmados e taxa de positividade entre as mesorregiões geográficas do estado do Paraná, durante o período de 1977 a 2012

\begin{tabular}{|c|c|c|c|c|c|c|}
\hline $\begin{array}{l}\text { Mesorregião } \\
\text { geográfica }\end{array}$ & $\begin{array}{c}\text { Total de } \\
\text { municípios }\end{array}$ & $\begin{array}{l}\text { Municípios com } \\
\text { registro de raiva }\end{array}$ & $\begin{array}{c}\text { Difusão da raiva entre } \\
\text { os municípios (\%) }\end{array}$ & $\begin{array}{l}\text { Amostras } \\
\text { colhidas }\end{array}$ & $\begin{array}{c}\text { Casos } \\
\text { confirmados }\end{array}$ & $\begin{array}{c}\text { Taxa de } \\
\text { positividade }(\%)\end{array}$ \\
\hline Centro Oriental & 14 & 14 & 100 & 2129 & 789 & 37,06 \\
\hline Curitiba & 37 & 32 & 86,49 & 2452 & 732 & 29,85 \\
\hline Norte Pioneiro & 46 & 32 & 69,57 & 1543 & 392 & 25,41 \\
\hline Oeste & 50 & 33 & 66,00 & 807 & 305 & 37,79 \\
\hline Norte Central & 79 & 22 & 27,85 & 827 & 148 & 17,9 \\
\hline Sudeste & 21 & 10 & 47,62 & 466 & 122 & 26,18 \\
\hline Noroeste & 61 & 22 & 36,07 & 558 & 74 & 13,26 \\
\hline Sudoeste & 37 & 12 & 32,43 & 265 & 69 & 26,04 \\
\hline Centro Sul & 29 & 11 & 37,93 & 395 & 46 & 11,65 \\
\hline Centro Ocidental & 25 & 2 & 8,00 & 104 & 5 & 4,81 \\
\hline TOTAL & 399 & 190 & 47,62 & 9546 & 2682 & 28,09 \\
\hline
\end{tabular}


demonstrando que há mesorregiões onde a chance de raiva é maior dentre os herbívoros acometidos por síndromes neurológicas (Centro Oriental e Oeste) e mesorregiões onde esta relação é menor (Noroeste, Centro Ocidental e Centro Sul).

Observou-se importância diferenciada de algumas mesorregiões na casuística da enfermidade. As mesorregiões Centro Oriental e de Curitiba foram as que apresentaram as ocorrências maiores (Fig.5) e juntas foram responsáveis por $56,7 \%$ de todos os casos do Paraná neste estudo.
Em conjunto com as mesorregiões Norte Pioneiro e Oeste, somaram-se $82,6 \%$ dos casos. Nessas quatro mesorregiões, e particularmente nas duas primeiras, a raiva assume, portanto, grande importância epidemiológica. Situação inversa poderia ser afirmada sobre as mesorregiões Noroeste, Centro Ocidental, Sudoeste e Centro Sul, que, juntas, albergaram apenas $7,1 \%$ dos casos durante o período. A variabilidade regional de ocorrência dentro de um mesmo estado é comum e já foi observada em levantamentos realizados no RJ (Feital \& Confalonieri 1998), em MG (Silva

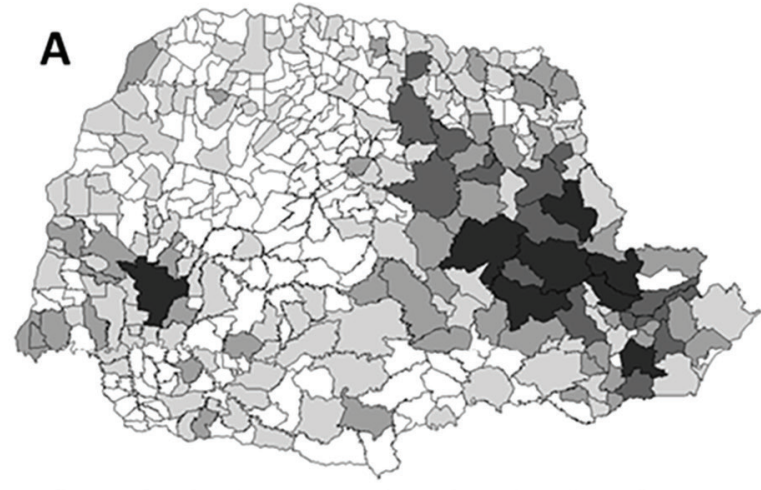

Ocorrência de casos de raiva dos herbivoros
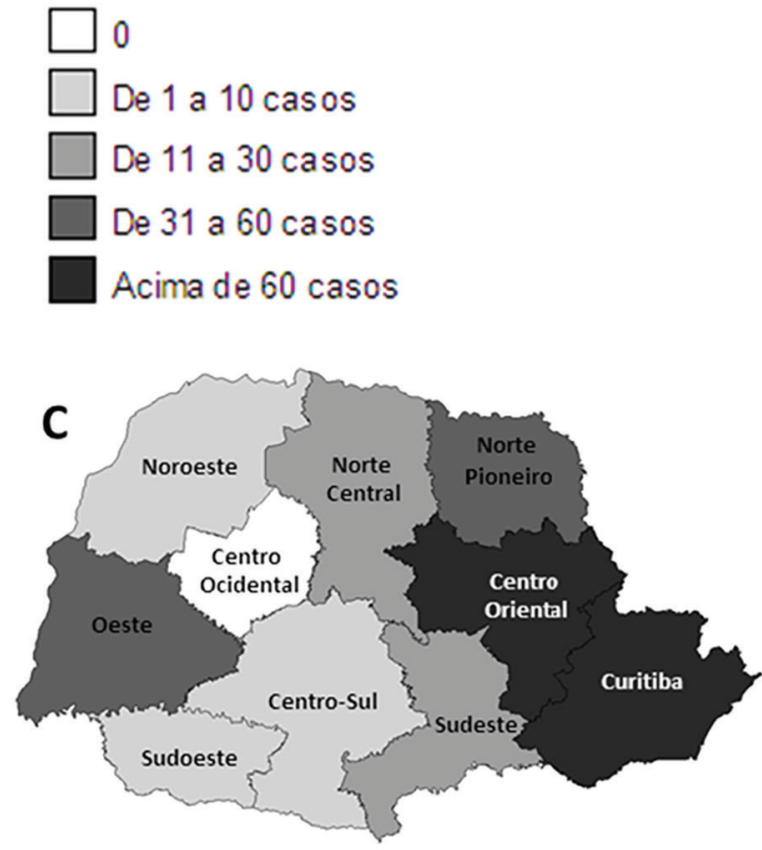

Ocorrência de casos de raiva dos herbívoros

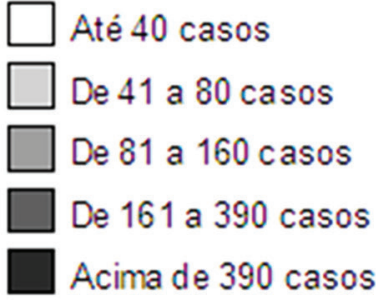

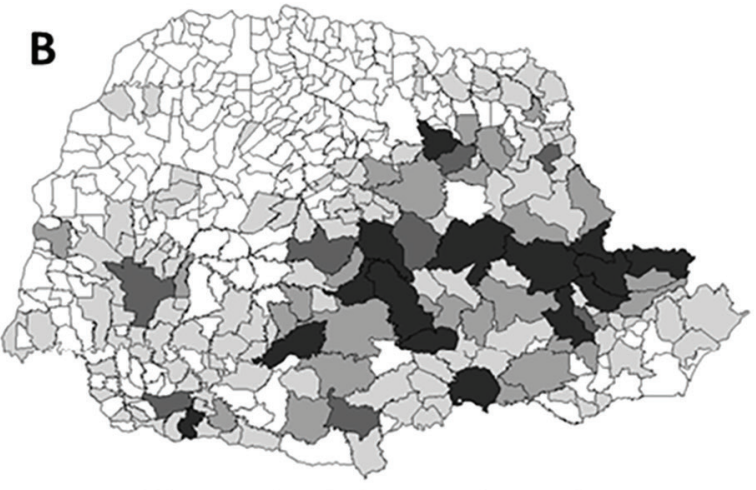

Número de abrigos cadastrados
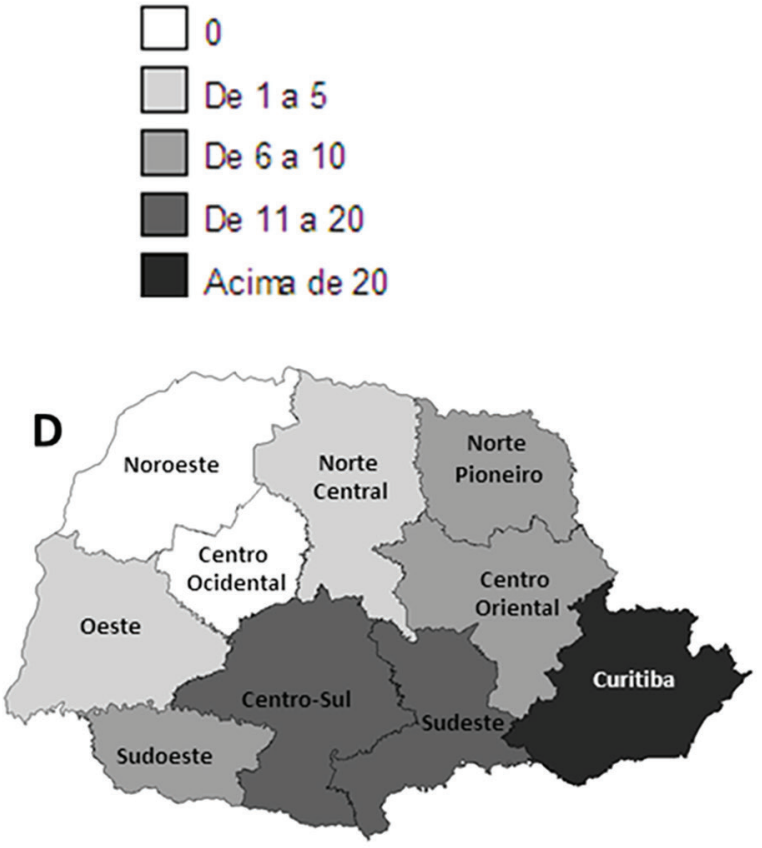

Número de abrigos cadastrados até 20

\section{De 21 a 70}

De 71 a 120

De 121 a 170

Acima de 170

Fig.5. (A) Distribuição espacial da raiva dos herbívoros e de abrigos conhecidos e monitorados de quirópteros hematófagos no estado do Paraná: ocorrência de casos por município, (B) número de abrigos por município, (C) ocorrência de casos por mesorregião geográfica e (D) número de abrigos por mesorregião geográfica. 
et al. 2001b), em MT (Matta et al. 2010) e em SP (Gomes \& Monteiro 2011). Foram estudadas associações deste fato com fatores ambientais (Silva et al. 2001a, Matta et al. 2010, Gomes \& Monteiro 2011), com o tipo de ocupação da terra e a população do rebanho bovino (Silva et al. 2001b, Gomes et al. 2011), com as ações de vigilância epidemiológica desempenhadas (Feital \& Confalonieri 1998, Menezes et al. 2008) e com as alterações de ecossistemas provocadas pelo homem (Feital \& Confalonieri 1998, Menezes et al. 2008).

Nesse estudo, verificou-se que a distribuição geográfica da raiva apresentou compatibilidade razoável com a distribuição dos abrigos de quirópteros hematófagos conhecidos e monitorados pelo estado (Fig.5), indicando que condições ambientais e/ou artificiais que propiciem albergue aos transmissores estão envolvidas com a ocorrência da enfermidade. É relevante citar que dentre os abrigos cadastrados, considerados neste estudo, 70,9\% eram naturais (cavernas, grutas, furnas, áreas ocas em tronco de árvores) e $29,1 \%$ eram artificiais (galerias pluviais e fluviais, edificações abandonadas, minas abandonadas, pontes e viadutos), demonstrando a relevância das alterações provocadas pelo homem no ambiente e a sua interação com o morcego transmissor.

No caso do Paraná, as mesorregiões onde houve maior número de casos (Centro Oriental e Curitiba) não são as mesmas onde se concentra, historicamente, o maior contingente de bovinos e de equinos do estado (mesorregiões Noroeste e Norte Central) (Paraná 2013), demonstrando que o efetivo do rebanho, por si só, não é fator diretamente ligado ao número de casos. A mesorregião de Curitiba, por exemplo, possui o menor rebanho de bovinos e de equídeos do estado. Por outro lado, possui o maior número de abrigos de morcegos hematófagos (209/940; 22,2\% de todos os abrigos conhecidos). Assim, sugere-se que o mosaico de ocorrência da enfermidade no PR sofre influência de condições que facilitem a fixação de abrigos pelos morcegos hematófagos. Considerando-se as 10 mesorregiões, o número de casos de raiva confirmados entre 2007 e 2012 não se correlacionou com o número de abrigos conhecidos e monitorados $(r=0,433 ; p>0,05)$. Porém, a correlação foi comprovada $(\mathrm{r}=0,469 ; \mathrm{p}<0,0001)$, ainda que fraca, quando se consideraram os municípios.

Levando em consideração os municípios individualmente, destacaram-se Castro (229 casos) e Tibagi (132 casos), na mesorregião Centro Oriental, e São José dos Pinhais (83 casos), Cerro Azul (63 casos) e Rio Branco do Sul (63 casos), na mesorregião de Curitiba. Nas mesorregiões Norte Pioneiro e Oeste, que concentraram número de casos logo abaixo daquele das mesorregiões já mencionadas (Fig.5), destacaram-se os municípios de Wenceslau Braz (49 casos) e de Figueira (40 casos) pelo Norte Pioneiro e de Cascavel
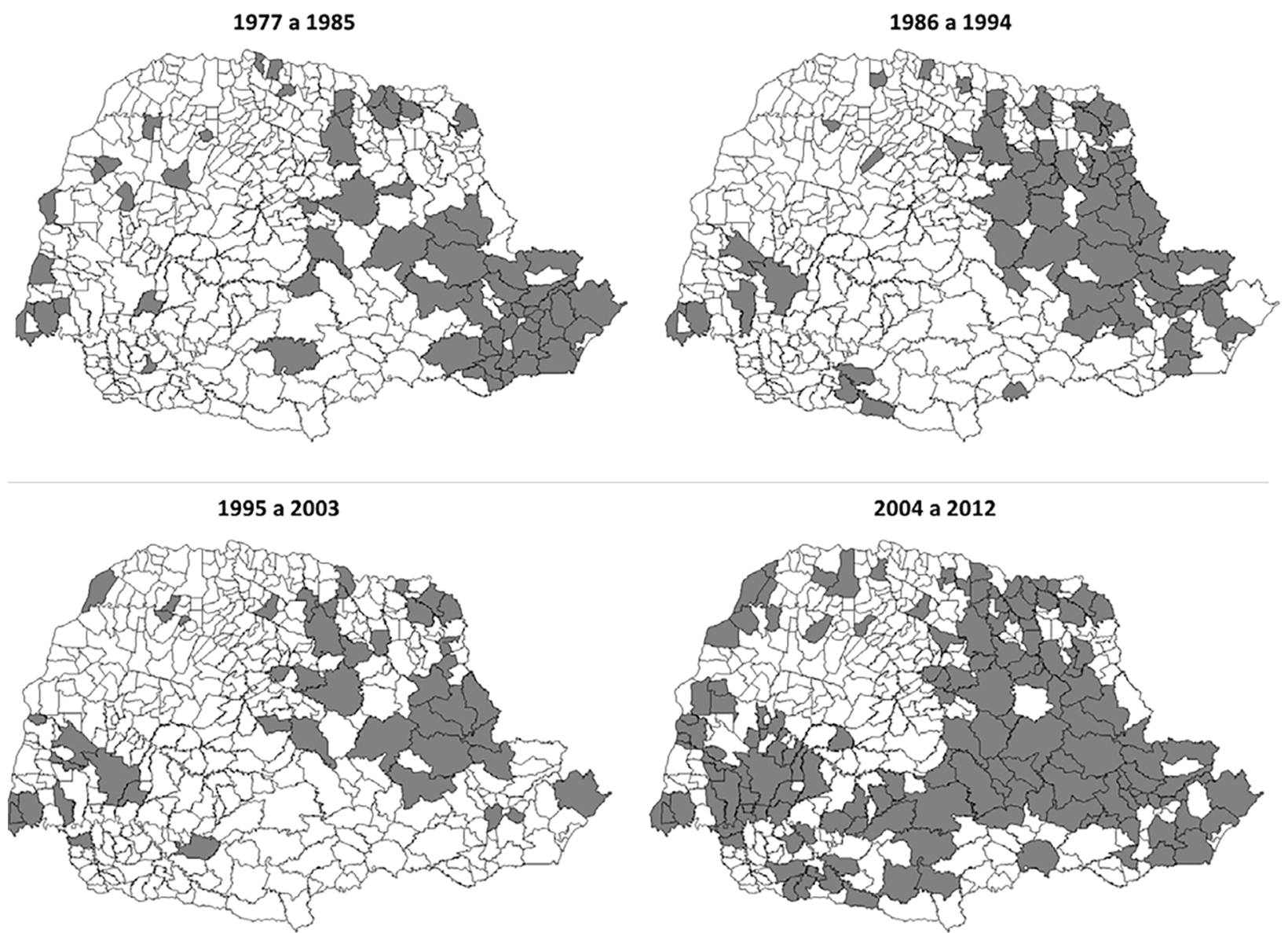

Fig.6. Progressão da ocorrência de casos de raiva dos herbívoros em municípios do estado do Paraná, dividida por diferentes períodos de anos, entre 1977 e 2012. 
(107 casos) pela região Oeste. Historicamente, observa-se que a progressão da enfermidade pelos municípios do estado teve períodos de situação estacionária e período de expansão (Fig.6). Esta característica também foi encontrada por Silva et al. (2001a) e por Menezes et al. (2008) em MG. Nos municípios das mesorregiões Centro Oriental, de Curitiba e Norte Pioneiro, e em alguns da mesorregião Oeste, a raiva vem ocorrendo desde o início do período de observação e manteve-se presente. De forma geral, a situação não parece muito distinta quando se consideram os três primeiros intervalos de nove anos (1977-2003). No período entre 2004 e 2012, houve a maior disseminação da doença, adentrando áreas em que anteriormente não havia registros da enfermidade e, ao mesmo tempo, mantendo-se em áreas nas quais a ocorrência era tradicional.

Esse fato também foi relatado em um levantamento realizado em MS (Neves 2008), onde houve, inclusive, inversão das áreas tradicionais de ocorrência, o que não parece estar acontecendo no PR já que os municípios das mesorregiões Centro Oriental e de Curitiba permanecem com número elevado de casos em praticamente todos os intervalos de tempo analisados. A expansão da raiva observada na última década, no PR, pode ter relação com a expansão geográfica dos abrigos de morcegos hematófagos. Isso é particularmente verdadeiro para as mesorregiões Sudeste, Centro Sul e Sudoeste (Fig.5), que passaram a sediar casos da doença somente no intervalo de anos mais recente (Fig.6). Nas mesorregiões Centro Sul e Sudoeste a grande maioria dos abrigos do Desmodus rotundus era natural $(93,8 \%$ e $78,5 \%$, respectivamente). Na mesorregião Sudeste a distribuição foi mais equilibrada (57,8\% eram naturais e $42,2 \%$ eram artificiais) sugerindo maior impacto das ações do homem sobre o ambiente. A observação de casos de raiva em maior número de municípios na última década pode estar relacionada também à maior efetividade das ações de vigilância epidemiológica para a doença executadas pelo serviço veterinário oficial.

É importante salientar que os dados desse estudo se referem unicamente aos casos notificados e que, por esse motivo, foram submetidos a método laboratorial para a confirmação do diagnóstico. Sabe-se que a subnotificação é uma condição comum em todo o território nacional, e o Paraná não é a exceção. 0 motivo para que essa situação exista e se mantenha tem relação com o comportamento do produtor rural que costuma convocar o serviço veterinário, autônomo ou oficial, somente após a morte seguida de três ou mais de seus animais. Além disso, após o diagnóstico confirmado em uma propriedade, geralmente os próximos casos contemporâneos de doença neurológica são assumidos também como raiva e a notificação não é feita. Por esses motivos, o número verdadeiro de casos de raiva dificilmente é conhecido e admite-se que para cada caso confirmado da doença, 10 outros ocorram e não sejam diagnosticados (Kotait et al. 1998).

Falhas eventuais no serviço de vigilância para a doença também podem ter contribuído para o número subestimado de casos confirmados. Especialmente nas mesorregiões Centro Ocidental, Centro Sul e Sudoeste, nas quais se registraram as menores taxas de ocorrência e a menor quanti- dade de amostras colhidas, esse fator de interferência pode ter exercido influência sobre os resultados. Nas mesorregiões Sudoeste e, principalmente, Centro Sul, o número reduzido de casos de raiva é discrepante com o número elevado de abrigos conhecidos de quirópteros. Na mesorregião Centro Ocidental, embora o número de abrigos seja pequeno, provavelmente porque as condições naturais não são favoráveis para o estabelecimento dos mesmos, chama atenção o percentual elevado de municípios nos quais a doença não foi registrada. De fato, não se pode descartar a possibilidade de que as ações de vigilância tenham sido pouco eficientes em algumas áreas do estado e isso pode ser particularmente verdadeiro no caso dos municípios que se apresentaram livres da doença.

A importância da raiva como doença neurológica que leva à morte, principalmente de bovinos e de equídeos, é evidente no PR. A participação do estado no panorama geral do Brasil é, contudo, pequena, contribuindo com somente 3,2\% de todos os casos ocorridos entre 1996 e 2010 no país (Brasil 2013). Em contrapartida, MG contribuiu com $33,2 \%$ dos casos no mesmo período, o que indica que a situação da enfermidade é ainda mais preocupante naquele estado. A relevância do Paraná, com relação à raiva, está bem representada dentro da Região Sul, sendo o estado que apresentou o maior número de casos notificados e responsável por $49,5 \%$ de todos os casos da região.

Esse é o primeiro levantamento realizado no estado do Paraná sobre a raiva dos herbívoros que abrange observações em um intervalo de tempo tão longo e que explora diferentes aspectos epidemiológicos envolvidos, incluindo a distribuição geográfica e a identificação das áreas de maior ocorrência da doença. Trata-se de uma doença endêmica que acomete, principalmente, bovinos e equídeos e ocorre durante todo o ano. Apresenta distribuição geográfica heterogênea com áreas em que a doença é tradicionalmente pouco frequente, áreas nas quais a raiva sempre esteve e continua presente, e áreas onde a doença passou a ocorrer na última década, caracterizando expansão geográfica. A compatibilidade geográfica entre a ocorrência da raiva e a localização dos abrigos do morcego Desmodus rotundus indica que esse é um importante fator associado com a doença. Os resultados desse levantamento indicam que o serviço oficial de defesa sanitária animal do PR vem desempenhando as ações de vigilância epidemiológica da raiva regularmente ao longo dos anos. Contudo, a diminuição da incidência da doença requer ações estratégicas mais efetivas. Como as medidas de controle direto da população do Desmodus rotundus são complexas e de execução mais difícil porque envolvem captura e uso seletivo da pasta anticoagulante por pessoal técnico qualificado, sugere-se que a imunização anual de bovinos e equídeos passe a ser adotada com regularidade nas áreas de maior risco (mesorregiões Centro Oriental e de Curitiba) e que os produtores rurais sejam esclarecidos e encorajados a vacinar seus rebanhos nas áreas de risco intermediário (mesorregiões do Norte Pioneiro e Oeste).

Agradecimentos.- Júlio A.N. Lisbôa é bolsista de produtividade em pesquisa do CNPq. 


\section{REFERÊNCIAS}

Acha P.N. \& Szyfres B. 2003. Rabia, p.351-383. In: Acha P.N. \& Szyfres B. (Eds), Zoonosis y Enfermedades Transmisibles Comunes al Hombre y a los Animales: clamidiosis, rickettsiosis y virosis. $3^{\text {a }}$ ed. Organización Panamericana de la Salud, Washington.

Aguiar M.S. 2007. Subfamília Desmodontinae, p.39-43. In: Reis N.R., Perachchi A.L., Pedro W.A. \& Lima I.P. (Eds), Morcegos do Brasil. Universidade Estadual de Londrina, Londrina, PR.

Arruda R.C.N, Barçante T.A., Peconick A.P., Pereira S.M., Souza J.C.P., Sousa T.M. \& Barçante J.M.P. 2013. Captura de Desmodus rotundus em regiões de mata e manguezais do estado do Maranhão: um estudo longitudinal. Pesq. Vet. Bras. 33(5):571-574.

Brasil 1976. Ministério da Agricultura, Pecuária e Abastecimento. Portaria no 126, de 18 de março de 1976. Disponível em: <http://sistemasweb. agricultura.gov.br/sislegis/action/detalhaAto.do?method=consultarLe gislacaoFederal> Acessado 15 set. 2014.

Brasil 2002. Ministério da Agricultura, Pecuária e Abastecimento. Instrução Normativa no 5, de 1o de março de 2002. Disponível em: <http:// sistemasweb.agricultura.gov.br/sislegis/action/detalhaAto.do?method $=$ consultarLegislacaoFederal $>$ Acessado 15 set. 2014 .

Brasil 2009. Ministério da Saúde. Guia de vigilância epidemiológica/ Ministério da Saúde, Secretaria de Vigilância em Saúde. 7ạ ed. Brasília. 842p.

Brasil 2013. Ministério da Agricultura, Pecuária e Abastecimento, Boletim de Defesa Sanitária Animal, Dados sobre a Raiva dos Herbívoros e Suínos no Brasil. Disponível em: <http://www.agricultura.gov.br/arq editor/file/Aniamal/programa\%20nacional\%20dos\%20herbivoros/ DADOS\%20RAIVA\%20ATE\%20SET\%202011.pdf> Acessado 30 mar. 2013.

Casseb L.M.N., Barbosa T.F.S., Pereira A.S., Vieira C.A., Medeiros D.B.A., Vasconcelos P.F.C., Travassos da Rosa E.S. \& Casseb A.R. 2006. Prevalência de raiva animal em amostras procedentes da região norte do Brasil, diagnosticadas no instituto Evandro Chagas no período de 2000 a 2004. Revta Ciênc. Agrárias 46:261-274.

Feital A.S.S. \& Confalonieri U.E.C. 1998. Estudo epidemiológico da raiva bovina no estado do Rio de Janeiro, Brasil (1980-1992). Revta Bras. Ciênc. Vet. 5(1):21-27.

Galiza G.J.N., Silva M.L.C.R., Dantas A.F.M., Simões S.V.D. \& Riet-Correa F. 2010. Doenças do sistema nervoso central de bovinos no semiárido nordestino. Pesq. Vet. Bras. 30(3):267-276.

Gomes M.N. \& Monteiro A.M.V. 2011. Raiva bovina no estado de São Paulo e sua distribuição espacial entre 1992 e 2003. Arq. Bras. Med. Vet. Zootec. 63(2):279-286.

Gomes M.N., Monteiro A.M.V. \& Escada M.I.S. 2011. Raiva bovina segundo os mosaicos de uso e cobertura da terra no estado de São Paulo entre 1992 e 2003. Arq. Bras. Med. Vet. e Zootec. 63(2):287-295.

Guedes K.M.R., Riet-Correa F., Dantas A.F.M., Simões S.V.D., Neto E.G.M., Nobre V.M.T. \& Medeiros R.M.T, 2007. Doenças do sistema nervoso central em caprinos e ovinos no semi-árido. Pesq. Vet. Bras. 27(1):29-38.

Lima E.F., Riet-Correa F., Castro R.S., Gomes A.A.B. \& Lima F.S. 2005. Sinais clínicos, distribuição das lesões no sistema nervoso e epidemiologia da raiva em herbívoros na região Nordeste do Brasil. Pesq. Vet. Bras. 25(4):250-264.

Kotait I., Gonçalves C.A., Peres N.F., Souza M.C.A.M. \& Tarqueta M.C. 1998. Controle da Raiva dos Herbívoros. Instituto Pasteur, São Paulo. 15p.

Marcolongo-Pereira C., Sallis E.S.V., Grecco F.B., Raffi M.B., Soares M.P. \& Schild A.L. 2011. Raiva em bovinos na região sul do Rio Grande do Sul: Epidemiologia e diagnóstico imuno-histoquimico. Pesq. Vet. Bras. 31(4):331-335.

Matta G.C.A., Nociti A.A.B., Carvalho A.A.B., Nociti R.P. \& Samara S.I. 2010. Caracterização epidemiológica da raiva bovina no estado de Mato Grosso, Brasil, no período de 1996 a 2006. Arqs Inst. Biológico, São Paulo, 77(4):601-607.

Medronho R.A., Werneck G.L. \& Perez M.A. 2009. Distribuição das doenças no espaço e no tempo, p.83-102. In: Medronho R.A. (Ed.), Epidemiologia. $2^{\underline{a}}$ ed. Atheneu, São Paulo.
Menezes F.L., Silva J.A., Moreira E.C., Meneses J.N.C., Magalhães D.F., Barbosa A.D. \& Oliveira C.S.F. 2008. Distribuição espaço-temporal da raiva bovina em Minas Gerais, 1998 a 2006. Arq. Bras. Med. Vet. Zootec. 60(3):566-573.

Morettin P.A. \& Toloi C.M.C. 2006. Análise de Séries Temporais. Editora Edgard Blücher, São Paulo. 538p.

Montaño J.A., Polack G.W. \& Mora E.F. 1987. Raiva bovina em animais vacinados. II. Situação epidemiológica no estado do Paraná, Brasil, 1984. Arq. Biol. Tecnol. 30(2):367-380.

Monteiro J.M., Preto A.A., Sprada E.R. \& Germano P.M.L. 1992. Perfil da raiva animal em Curitiba e área metropolitana durante o período de 1955 a 1989. Arq. Biol. Tecnol. 35(1):23-35.

Mori A.E. \& Lemos R.A.A. 1998. Raiva. p.47-58. In: Lemos R.A.A. (Ed.), Principais Enfermidades de Bovinos de Corte do Mato Grosso do Sul: reconhecimento e diagnóstico. UFMS, Campo Grande.

Neves D.A. 2008. Sazonalidade e ciclicidade da raiva em herbívoros domésticos no estado do Mato Grosso do Sul, 1998 a 2006. Dissertação de Mestrado em Ciência Animal, Faculdade de Medicina Veterinária e Zootecnia, Universidade Federal do Mato Grosso do Sul, Campo Grande, MS. 84p.

Office International des Epizooties 2014. Rabies: manual of diagnostic tests and vaccines for terrestrial animals. 7th ed. OIE. 1404p. Disponível em: <http://www.oie.int/fileadmin/Home/eng/Health_standards/ tahm/2.01.13_RABIES.pdf> Acessado 9 fev. 2014.

Oliveira T.S., Bull V., Rezende C.A., Furtini R., Costa E.A., Paixão T.A. \& Santos R.L. 2012. Perfil das amostras do sistema nervoso central de bovinos com síndrome neurológica e diagnóstico da raiva bovina no serviço de defesa sanitária de Minas Gerais, 2003-2010. Pesq. Vet. Bras. 32(4):333339.

Paraná 2013. Instituto Paranaense de Desenvolvimento Econômico e Social, Consultas/Agropecuária/Pecuária e Aves. Disponível em <http:// www.ipardes.pr.gov.br/imp/index.php> Acessado 1 jul. 2013.

Patrício M.A.C., Dittrich R.L., Sponchiado D., Richartz R.R. \& Barros Filho L.R. 2007. Prevalência da raiva em bovinos, ovinos e caprinos no estado do Paraná. Arch. Vet. Sci. 12(Supl.):267-268.

Patrício M.A.C., Richartz R.R.T.B., Willig F.H., Sponchiado D., Dittrich R.L. \& Barros Fillho I.R. 2009. Prevalência da raiva em bovinos, ovinos e caprinos no ano de 2007 no estado do Paraná. Cienc. Anim. Bras. 10(Supl.):519-524.

Pimentel L.A., Oliveira D.M., Galiza G.J.N., Rego R.O., Dantas A.F.M. \& Riet-Correa F. 2009. Doenças do sistema nervoso central de equídeos no semi-árido. Pesq. Vet. Bras. 29(7):589-597.

Queiroz H.Q., Carvalho C., Buso D.S., Ferrari C.I.L. \& Pedro W.A. 2009. Perfil epidemiológico da raiva na região noroeste do estado de São Paulo no período de 1993 a 2007. Revta Soc. Bras. Med. Trop. 42(1):9-14.

Queiroz G.R., Oliveira R.A.M. \& Lisbôa J.A.N. 2013. Doenças do sistema nervoso dos bovinos no Estado do Paraná diagnosticadas entre 2009 e 2012. Revta Conselho Regional de Medicina Veterinária do Paraná 12(39):28-30.

Ribas N.L.K.S., Carvalho R.I., Santos A.C., Valençoela R.A., Gouveia A.F., Castro M.B., Mori A.E. \& Lemos R.A.A. 2013. Doenças do sistema nervoso de bovinos no Mato Grosso do Sul: 1082 casos. Pesq. Vet. Bras. 33(10):1183-1194.

Rissi D.R., Fighera R.A., Irigoyen L.F., Kommers G.D. \& Barros C.S.L. 2010. Doenças neurológicas de ovinos na região Central do Rio Grande do Sul. Pesq. Vet. Bras. 30(3):222-228.

Rodriguez L.L., Rohe P.M., Batista H. \& Kurath G. 2007. Rhabdoviridae, p.691-718. In: Flores E.F. (Ed.), Virologia Veterinária. UFSM, Santa Maria.

Sanches W.D., Langohr I.M., Stigger A.L. \& Barros C.S.L. 2000. Doenças do sistema nervoso central em bovinos no sul do Brasil. Pesq. Vet. Bras. 20(3):113-118.

Santos M.F.C., Resende R.M., Sobrinho R.N. \& Vieira S. 2006. Diagnóstico laboratorial da raiva no estado de Goiás no período de 1976 a 2001. Ciênc. Anim. Bras. 7(1):77-83.

Schlögel F. 1985. Breve histórico da raiva. Arq. Biol. Tecnol. 28(2):277-312. Silva J.A., Moreira E.C., Haddad J.P.A., Modena C.M. \& Tubaldini M.A.S. 
2001a. Distribuição temporal e espacial da raiva bovina em Minas Gerais, 1976 a 1997. Arq. Bras. Med. Vet. Zootec. 53(1):1-11.

Silva J.A., Moreira E.C., Haddad J.P.A., Sampaio I.B.M., Modena C.M. \& Tubaldini M.A.S. 2001b. Uso da terra como determinante da distribuição da raiva bovina em Minas Gerais, Brasil. Arq. Bras. Med. Vet. Zootec. 53(3):273-283.

Teixeira T.F., Holz C.L., Caixeta S.P.M.B., Dezen D., Cibulski S.P., Silva J.R., Rosa J.C.A., Schmidt E., Ferreira J.C., Batista H.B.C.R., Caldas E., Franco
A.C. \& Roehe P.M. 2008. Diagnóstico de raiva no Rio Grande do Sul, Brasil, de 1985 a 2007. Pesq. Vet. Bras. 28(10):515-520.

Uieda W. 1982. Aspectos do comportamento alimentar das três espécies de morcegos hematófagos (Chiroptera, Phyllostomidae). Dissertação de Mestrado em Biologia, Universidade Estadual de Campinas, Campinas, SP. 166p.

Wada M.Y., Rocha S.M. \& Maia-Elkhoury A.N.S. 2011. Situação da raiva no Brasil, 2000 a 2009. Epidemiol. Serv. Saúde 20(4):509-518. 